\title{
In-Situ Tensile Deformation of Additively Manufactured Ti 6Al 4V
}

\author{
John R. Porter ${ }^{1}$, Robert Wheeler ${ }^{1}$ and Michael Velez ${ }^{1}$ \\ ${ }^{1 .}$ UES Inc., Dayton, USA.
}

Ti $6 \mathrm{Al} 4 \mathrm{~V}$ is the most mature alloy currently used in the manufacture of parts by the Electron Beam Melting (EBM) additive manufacturing (AM) process. EBM is a powder bed process in which parts are built up, layer by layer, by using an electron beam scanned over the powder bed in a raster that defines a given layer of the part. The electron beam melts a pool of material that resolidifies as the probe traverses the layer. Typically, each layer is between $50 \mu \mathrm{m}$ and $70 \mu \mathrm{m}$ thick. During resolidification, the underlying metal grows epitaxially such that mm long, [100 $]_{\mathrm{c}}$ oriented columnar grains can develop with the columnar grains growing parallel to the build direction. Such columnar grains can clearly be seen in Fig. 1A.

After cooling from the build temperature, $\mathrm{Ti} 6 \mathrm{Al} 4 \mathrm{~V}$ is an alpha/beta alloy that initially solidifies as cubic beta phase that mostly transforms on further cooling to hexagonal alpha phase. The well established $(110)_{\mathrm{c}} / /(0001)_{\mathrm{h}}$ orientation relationship results in a Widmanstatten microstructure with 12 variants of alpha phase within each prior beta columnar grain. The Widmanstatten microstructure is depicted in Figure 1B.

While the AM as-built microstructure is well characterized and its formation well understood, the goal of an equiaxed prior beta structure has not been realized. The question then presents itself as to whether the prior beta grain boundaries represent planes of weakness. Here we address this by undertaking a series of in-situ micromechanical tests in the SEM. Microsamples were prepared that were oriented longitudinally within one prior beta grain, transversely within one prior beta grain and transversely across one prior beta boundary.

Tests were conducted using a Microtest in-situ test rig. Samples were FIB cut from previously prepared electropolished TEM foils selected from regions of the foil that were $\sim 10 \mu \mathrm{m}$ thick. After cutting the tensile bar outline, the samples were FIB trimmed to have flat and parallel opposing sides. The tensile gauge after preparation was approximately $100 \mu \mathrm{m}$ x $40 \mu \mathrm{m}$ x $10 \mu \mathrm{m}$ thick.

Figure 3 shows a sequence of images taken in the unloaded condition and just prior to failure. Fracture is believed to have happened at a prior beta grain boundary.

When tested parallel to the growth direction, the sample showed a very different fracture behavior. The sample necked and the supported load dropped. The test was interrupted prior to failure for further microstructural analysis.

References:

[1] B. Saller et al., Microscopy and Microanalysis 16 (Suppl. 2), (2010) p. 702.

[2] J. R. Porter et al., Materials Science and Technology (MS\&T) (2011) p. 1434.

[3] P. A. Shade et al., Acta Materiala 57 (2009) p. 4580.

[4] The authors acknowledge funding from the AFRL under contract FA8650-14-C-5021 

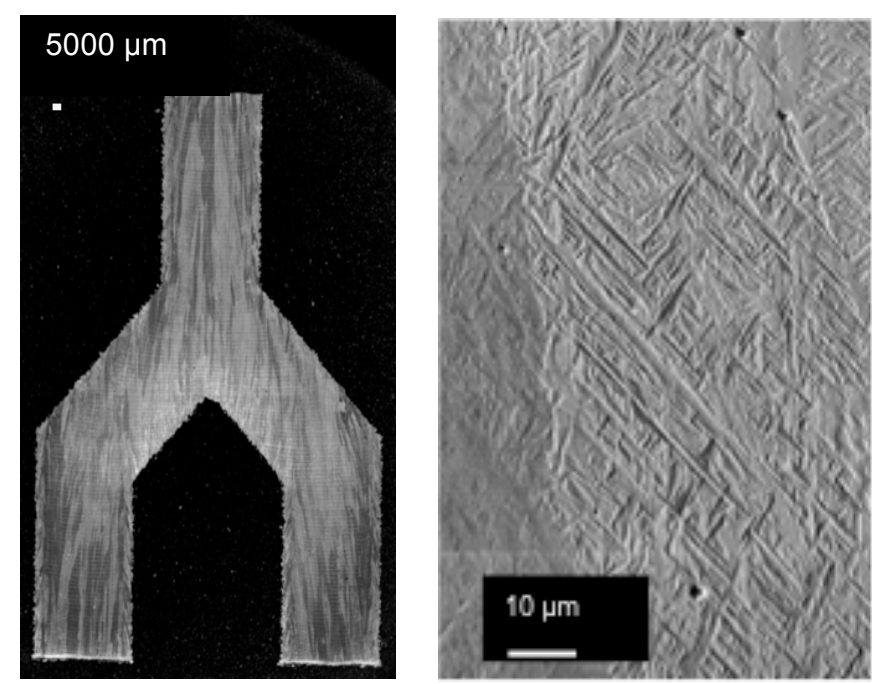

Figure 1. (a) Tuning fork part grown by EBM revealing columnar prior beta grain structure. (b) Widmanstatten structure of the transformed alpha within the prior beta grains.
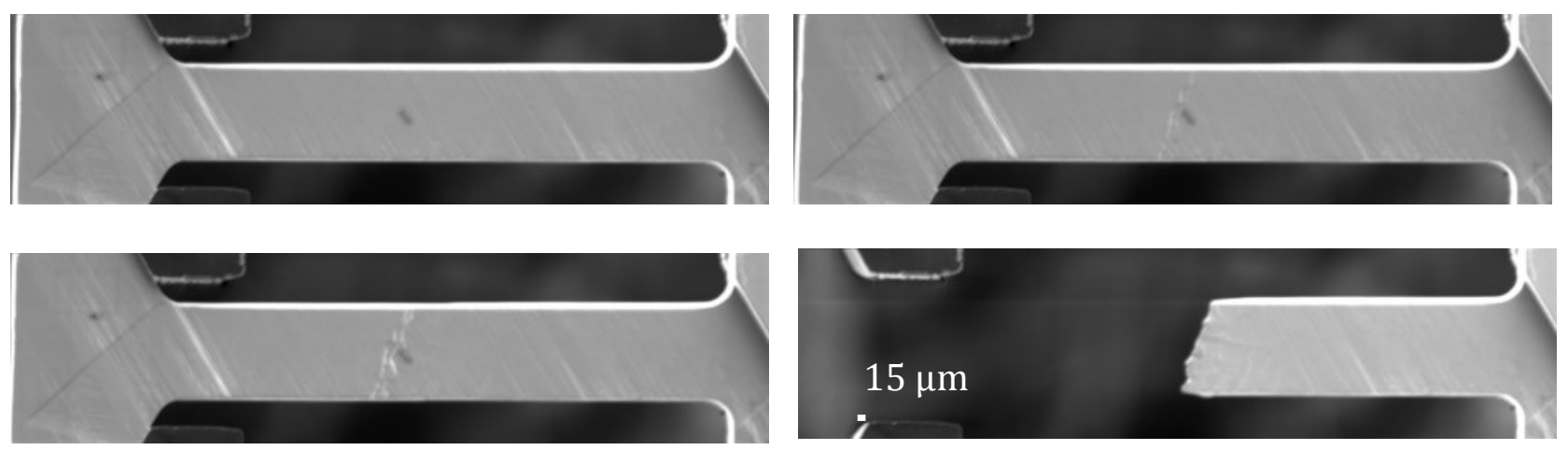

Figure 2. Sequence of images taken during micromechanical test just prior to failure. Transverse sample spanned a prior beta grain boundary.
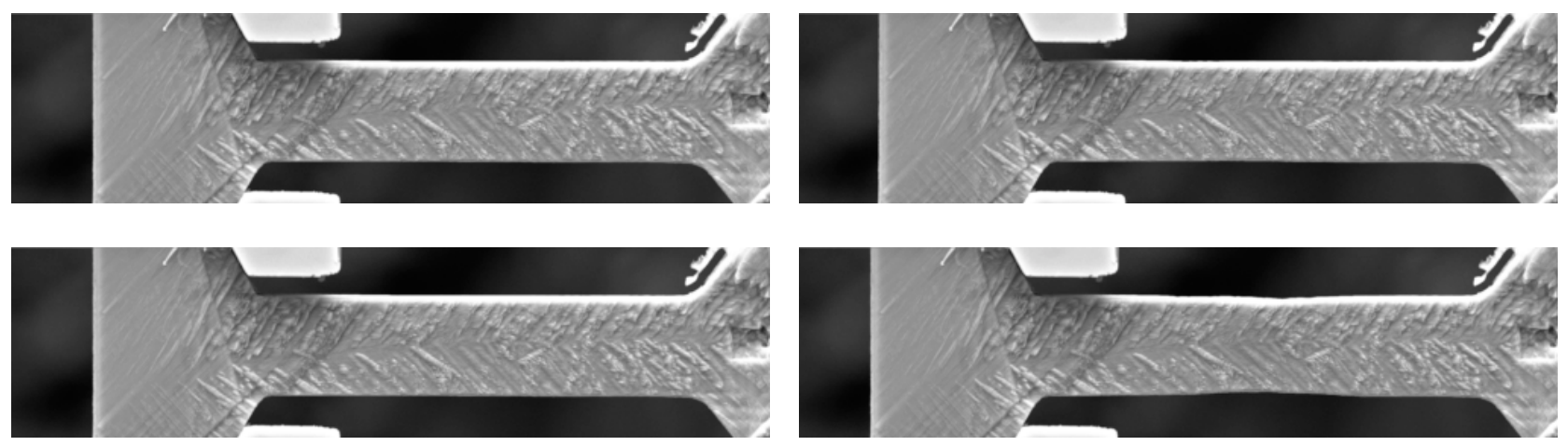

Figure 3. Sequence of images taken during micromechanical test just prior to yield. Build direction sample included a prior beta grain boundary parallel to the sample. 